\title{
A Experiência de Melhoria do Processo do Instituto Atlântico Baseado no SW-CMM nível 2
}

\author{
Carlos Giovano Pires, Fabiana Marinho, Gabriela Telles, Arnaldo Belchior * \\ Instituto Atlântico, Rua Chico Lemos, 946, 60822-780, Fortaleza - Ceará \\ * Universidade de Fortaleza, Mestrado em Informática Aplicada, \\ Av. Washington Soares, 1321, 60.811-341, Fortaleza-Ceará \\ \{cgiovano, fabiana, gabi\}@atlantico.com.br, belchior@unifor.br
}

\begin{abstract}
Resumo
Este trabalho relata a experiência de planejamento e implantação do SW-CMM nível 2 no Instituto Atlântico. Este processo foi planejado com base no ciclo PDCA e realizado de acordo com as seguintes etapas: planejamento, diagnóstico do processo existente, definição de novos processos, implantação e uso dos processos definidos, e avaliações. Como principais resultados obtidos, ressaltamos o aumento na qualidade dos produtos entregues aos clientes e amadurecimento do processo de desenvolvimento da instituição.

Palavras-chave: melhoria de processo de software, CMM
\end{abstract}

\begin{abstract}
This work describes the Instituto Atlântico experience of planning and implementation of the SW-CMM level 2. This process was planned based on PDCA's cicle and executed in accordance with the following phases: planning, diagnosis of the existing process, definition of new processes, implementation and use of the defined processes, and assessments. The main results of this experience was the increase in the product quality delivered to the customers and the maturity of the institution development process.
\end{abstract}

Keywords: improvement software process, CMM

\section{Introdução}

A globalização da economia, a rápida evolução da tecnologia da informação e o movimento irreversível da qualidade estão alavancando o processo de reestruturação de conceitos, princípios e crenças, onde organizações bem sucedidas fundamentam suas estratégias e seus planejamentos, para assegurarem a vantagem competitiva no mercado [1].

O Capability Maturity Model for Software (SW-CMM) desenvolvido pelo Software Engineering Institute (SEI) é um modelo que descreve os elementos chave de um processo de software efetivo. O SW-CMM descreve um caminho de melhoria contínua para empresas de desenvolvimento de software, a partir de um processo imaturo para um processo maturo e disciplinado [2].

Este trabalho descreve a experiência do Instituto Atlântico (IA) na implantação do SW-CMM nível 2 e apresenta os resultados obtidos com a melhoria de seu processo de software. O Instituto Atlântico é uma instituição de pesquisa e desenvolvimento localizada em Fortaleza (CE), fundada em novembro de 2001 por iniciativa do Centro de Pesquisa e Desenvolvimento em Telecomunicações (CPqD). O IA desenvolve software em diversas áreas tecnológicas, posicionando-se como fonte inovadora de conhecimento e de geração de resultados. Sua missão é desenvolver e difundir tecnologias de alto valor agregado para o sucesso de seus clientes, através de relações duradouras com parceiros que resultem em benefícios para a sociedade. Baseado nos valores de inovação, excelência, comprometimento e respeito, o IA busca a melhoria contínua da qualidade de seus processos de software, visando o aumento de produtividade, o cumprimento de prazos estabelecidos, a redução de custos do produto final e, sobretudo, atender cada vez melhor a seus clientes.

O SW-CMM foi escolhido por se tratar de um modelo de qualidade desenvolvido especificamente para organizações de software, que buscam o controle de seus processos de 
desenvolvimento e uma cultura de excelência em engenharia de software e gerência de projetos. O SW-CMM cobre práticas de planejamento, engenharia e gerência de desenvolvimento de software. Quando seguidas, essas práticas melhoram a capacidade da organização para atingir objetivos de custo, prazo, funcionalidade e qualidade do produto. $\mathrm{O}$ CMM é um modelo de referência que define o que fazer, e não como fazer [5].

Este trabalho está organizado em 4 seções. A seção 2 descreve o perfil do Instituto Atlântico. O processo de implantação do SW-CMM nível 2 é apresentado na seção 3 . Na seção 4, foram analisados os resultados obtidos com a melhoria do processo de software no IA. Finalmente, a seção 5 contém as conclusões e os direcionamentos para trabalhos futuros.

\section{$2 \quad$ O Perfil do Instituto Atlântico}

O escopo de atuação do IA abrange pesquisa e desenvolvimento de soluções de software e hardware, e serviços de consultoria na área de tecnologia da informação (TI) e telecomunicações. Estas soluções são desenvolvidas mediante projetos próprios ou específicos para clientes, e podem ter como objetivo uma nova tecnologia, um novo produto ou atualização de algum já existente.

Com uma equipe de 107 colaboradores atuando em 11 projetos, o IA é considerado uma instituição de pequeno porte para os padrões internacionais. O Gráfico 1 mostra a evolução do crescimento da equipe do IA.

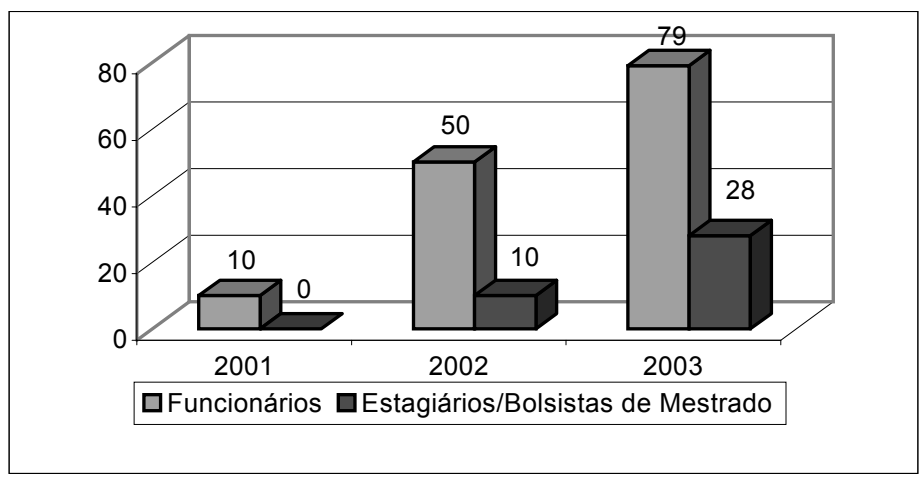

Gráfico 1: Evolução do crescimento da equipe do IA

Os projetos desenvolvidos pertencem a diversas áreas e possuem tamanhos variados. Algumas áreas de projeto são: simulação de redes $3 \mathrm{G}$ para sistemas celulares, sistemas de informação geográficos na Web, segurança da informação, integração de sistemas com XML, gerência de redes com SNMP, portais Web, ambientes de simulação para cabos, aplicações para dispositivos móveis e aplicações de transações bancárias. O tamanho médio dos projetos tem variado de 1.500 homens/hora a 20.000 homens/hora.

\section{O Processo de Implantação do SW-CMM Nível 2}

A implantação do SW-CMM nível 2 no IA foi planejada com base no ciclo PDCA [3] de modo a ocorrer de forma gradual, visando reduzir o impacto das mudanças necessárias nas práticas institucionais e respeitando sua cultura organizacional. As atividades realizadas durante a implantação foram agrupadas em cinco etapas: $(i)$ planejamento; (ii) diagnóstico do processo anterior; (iii) definição dos novos processos; (iv) implantação e uso dos processos definidos; e (v) avaliações. Cada uma dessas etapas está descrita a seguir. 


\subsection{Planejamento}

Nesta etapa, foi criado o grupo responsável pelo planejamento, pela gerência e execução das atividades de melhoria dos processos de software do IA denominado grupo de Suporte ao Processo de Desenvolvimento (SPD). Esse grupo era composto por seis pessoas, com alocação parcial. O cronograma contendo o planejamento das atividades a serem realizadas foi elaborado conforme mostrado na Tabela 1.

Tabela 1: Cronograma de atividades

\begin{tabular}{|c|c|}
\hline ETAPA / ATIVIDADE & PERÍODO \\
\hline \multicolumn{2}{|l|}{ Planejamento } \\
\hline Criação do SPD e elaboração do cronograma & Julho/02 \\
\hline \multicolumn{2}{|l|}{ Diagnóstico do processo anterior } \\
\hline Parceria com a UNIFOR e análise dos processos do CPqD & Julho/02 - Outubro/02 \\
\hline Oficina de divulgação dos resultados & Outubro/02 \\
\hline \multicolumn{2}{|l|}{ Definição dos novos processos } \\
\hline Início da consultoria externa e elaboração do plano de ações & Novembro/02 \\
\hline \multicolumn{2}{|l|}{ Implantação e uso dos processos definidos } \\
\hline Implantação dos processos nos projetos pilotos & Dezembro/02 - Fevereiro/03 \\
\hline Baseline dos novos processos e sua implantação em todos os projetos & Março/03 \\
\hline \multicolumn{2}{|l|}{ Avaliações } \\
\hline Diagnóstico da situação atual (mini avaliação) & Julho/03 \\
\hline Treinamento no método SCE (Software Capability Evaluation) & Agosto/03 \\
\hline Avaliação interna e avaliação independente de SQA & Setembro/03 \\
\hline Avaliação oficial SW-CMM nível 2 & Outubro/03 \\
\hline
\end{tabular}

\subsection{Diagnóstico do Processo Anterior}

Os processos e procedimentos do IA foram definidos com base nos procedimentos do CPqD, principal parceiro e agente criador do IA. Nesse momento, o CPqD também se encontrava em processo de melhoria de software buscando o SW-CMM nível 2. Os procedimentos foram analisados sob dois aspectos: (i) mapeamento para as práticas do SW-CMM nível 2; e (ii) adequação dessas práticas à realidade e ao contexto do IA.

Esse trabalho foi realizado pelo SPD juntamente com professores da Universidade de Fortaleza (UNIFOR), especialistas em qualidade de software. Nesta etapa, foram analisados os documentos dos processos de software existentes (procedimentos, planos, relatórios, modelos etc.) e os documentos elaborados pelos projetos (especificação de requisitos, plano de desenvolvimento etc.). Além disso, foram realizadas reuniões regulares do SPD com membros das equipes dos projetos e parceiros da UNIFOR.

Os resultados foram disponibilizados em um documento, contendo o grau de aderência do processo de software do IA ao SW-CMM nível 2, que foi apresentado em oficina com gerentes e coordenadores dos projetos.

\subsection{Definição de Novos Processos}

Nesta etapa, os processos diagnosticados foram adaptados e novos processos foram definidos de modo a contemplar os requisitos do SW-CMM nível 2 [6]. Na engenharia de software, um modelo de processo é um conjunto de passos parcialmente ordenados com a intenção de construir um produto de software de qualidade, capaz de atender às necessidades e exigências do usuário final de acordo com planejamento e orçamento previstos [4]. 
Devido à natureza essencialmente gerencial dos processos do SW-CMM nível 2 e ao porte da instituição, observou-se que a melhor estratégia seria definir um conjunto de procedimentos institucionais para todos os projetos contendo pontos e guias de adaptação. Desta forma, as melhores práticas e métricas seriam obtidas ao longo do processo de melhoria do SW-CMM nível 2, formando uma base sólida para o processo de melhoria do SW-CMM nível 3. É importante ressaltar que a estratégia de institucionalização dos procedimentos facilitou o trabalho de definição, implantação e suporte dos processos de software do IA.

Os novos processos passaram a integrar o sistema de qualidade do IA, sendo composto por políticas institucionais da qualidade, procedimentos, templates, registros e orientações, conforme mostrado na Figura 1. As políticas institucionais da qualidade definem as metas e diretrizes institucionais, a estrutura organizacional, e principais papéis e responsabilidades. Os procedimentos descrevem o processo de trabalho para cada área chave do SW-CMM nível 2 e detalham a execução das atividades do processo. Os templates são modelos de documentos que são foram gerados para auxiliar na execução das atividades descritas em cada procedimento. Os registros são os dados gerados pelos projetos ao longo do ciclo de vida. As orientações são guias para uso de ferramentas, tecnologias e atividades específicas do processo de desenvolvimento.

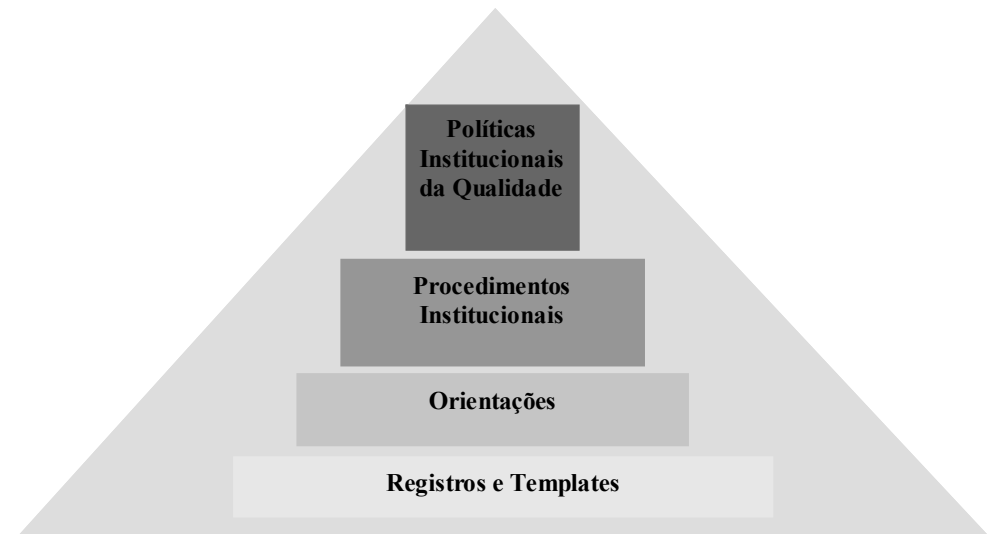

Figura 1: Sistema de qualidade do Instituto Atlântico

As atividades realizadas nesta etapa foram acompanhadas por uma consultoria externa de uma empresa especializada em CMM, certificada pelo SEI. Como resultado dessa consultoria foi gerado um plano de ação para adequação dos novos processos ao SW-CMM nível 2.

Posteriormente, os processos foram validados com os envolvidos das principais áreas chaves, através de oficinas. Os procedimentos foram apresentados pelo SPD e os participantes deram sugestões de melhoria e adequação dos mesmos às práticas dos projetos. Após as oficinas, os procedimentos ficaram mais aderentes à realidade dos projetos e os grupos envolvidos mostraram-se engajados com a implementação dos processos institucionalizados.

\subsection{Implantação e Uso dos Processos Definidos}

A implantação dos novos processos, juntamente com a atuação do SQA, ocorreu inicialmente, em três projetos pilotos. Essa estratégia foi adotada com o objetivo de avaliar os processos definidos com base nas atividades do grupo de SQA. Desta forma, ajustes necessários foram realizados, foi gerada uma baseline dos procedimentos institucionais, os membros das equipes de projetos foram treinados e os processos implantados em toda a organização. 
Neste momento, o SPD foi reestruturado para se tornar um grupo institucional com atuação nas áreas de qualidade, processos institucionais, testes, arquitetura e gestão de configuração.

O SPD passou a ser formado pelos seguintes subgrupos: grupo de gestão de configuração (GGC), grupo de testes (GrT), grupo de arquitetos (GrArq), grupo de garantia da qualidade (SQA) e grupo de processo de engenharia de software (SEPG). A formação e alocação do grupo está descrita na Tabela 2.

\begin{tabular}{|l|c|c|c|} 
GRUPO & $\begin{array}{c}\text { Tabela 2: Alocação dos Sub-grupos do SPD } \\
\text { PESSOAS ALOCADAS } \\
\text { DEDICAÇÃO TOTAL }\end{array}$ & $\begin{array}{c}\text { PESSOAS ALOCADAS } \\
\text { DEDICAÇÃO PARCIAL }\end{array}$ & $\begin{array}{c}\text { DEDICAÇÃO TOTAL } \\
\text { (HORAS/SEMANA) }\end{array}$ \\
\hline GGC & 0 & 2 & 16 \\
\hline GrArq & 1 & 1 & 60 \\
\hline GrT & 2 & 0 & 80 \\
\hline SQA & 2 & 1 & 100 \\
\hline SEPG & 0 & 3 & 24 \\
\hline
\end{tabular}

O GGC tem como objetivo principal apoiar tecnicamente os projetos nas atividades pertinentes à gestão de configuração. O GrArq apóia os projetos na definição de arquiteturas de sistemas e atividades de engenharia de produto. O GrT é responsável por definir procedimentos de verificação independente e executar análises sistêmicas de projetos de acordo com cronograma estabelecido, garantindo assim a entrega de produtos com qualidade e atendendo ao escopo definido pelo cliente. O grupo de SQA apóia e orienta projetos na utilização de práticas institucionais, avaliando-os quanto à conformidade com os procedimentos institucionais. O SEPG mantém e controla o sistema de qualidade do IA.

Durante a implantação dos processos, algumas dificuldades foram encontradas. Inicialmente, as equipes dos projetos mostraram-se resistentes à adoção dos novos processos e avaliações realizadas pelo grupo de SQA. Este problema foi solucionado com o envolvimento da alta gerência, que motivou as equipes por meio de palestras e oficinas. Além disso, o grupo de SQA direcionou suas atividades mais fortemente para a orientação da execução das novas atividades do processo.

Outro problema identificado foi uma resistência inicial dos projetos de pequeno porte em utilizar algumas práticas dos procedimentos institucionais. Para solucionar este problema, foi realizada uma adaptação dos procedimentos para possibilitar maior flexibilidade e pontos de adaptação para projetos de pequeno porte.

O rápido crescimento da instituição causou o ingresso freqüente de novos funcionários que não estavam treinados nas práticas e nos processos internos. Para solucionar esta questão, o IA gerou um treinamento chamado "Kit de Boas-Vindas" que abordava os conceitos de processos, CMM e processos internos do IA. Antes de ser alocado a um projeto, o novo funcionário era treinado pelo grupo de SQA. Além disso, foram identificadas pessoas, entre os funcionários que já trabalhavam na instituição, que desconheciam os procedimentos e as práticas a serem seguidas. Com o objetivo de minimizar as deficiências encontradas, o SEPG elaborou um cronograma de treinamentos para as principais áreas chave do processo dando ênfase aos problemas mais freqüentes encontrados pelo SQA. Esses problemas estão descritos na Tabela 3.

Tabela 3: Principais problemas encontrados na implantação do SW-CMM nível 2 ÁREA CHAVE Requisitos PRINCIPAIS PROBLEMAS

\begin{tabular}{ll} 
Planejamento & - Processo de alterações de requisitos \\
\hline Acompanhamento & - Estimativas de tamanho \\
\hline - Restimativas
\end{tabular}




$\begin{array}{ll}\text { - Acompanhamento dos tamanhos dos produtos } \\ \text { Configuração } & \text { - Planejamento das baselines } \\ & \text { - Registro de alteração de baseline } \\ \text { - Realização das auditorias de configuração } & \text { - Definição e coleta de indicadores }\end{array}$

A atuação do grupo de SQA foi de fundamental importância para a fase de institucionalização dos processos. No entanto, a tarefa de determinar qual a melhor forma de atuação do SQA, como este grupo deve ser estruturado e quantas horas deve disponibilizar para cada projeto é bastante complexa.

Quanto à forma de atuação, optamos inicialmente por conduzir avaliações com periodicidade de três semanas, focando todas as áreas-chave do SW-CMM nível 2 [6]. Foi identificado que, devido ao longo intervalo entre avaliações, alguns problemas surgiam e na avaliação seguinte não podiam mais sofrer ações corretivas, tornando-se desvios. A solução adotada para minimizar este problema foi a realização de avaliações semanais. No entanto, revisar todas as práticas no período de uma semana mostrou-se uma tarefa árdua e com retorno pouco expressivo. Por fim, o SQA passou a realizar avaliações semanais com base na fase atual do projeto, criando um mini-planejamento para identificar as práticas relevantes de acordo com as fases do projeto. Uma conseqüência interessante desta forma de atuação foi que, além da resolução rápida de problemas, o esforço de SQA alocado a cada projeto reduziu. Atualmente, trabalhamos com as métricas para planejamento de alocação de SQA apresentadas na Tabela 4.

Tabela 4: Média de esforço de SQA por porte de projeto PORTE DO PROJETO ESFORÇO EM HOMENS/HORAS

\begin{tabular}{l|l}
\hline Projeto Pequeno & 12 \\
\hline Projeto Médio & 30 \\
\hline Projeto Grande & 36
\end{tabular}

\subsection{Avaliações}

O processo de melhoria de software do IA foi iniciado em julho de 2002. Em agosto de 2003 foi realizada uma mini-avaliação. $\mathrm{O}$ escopo da mini-avaliação foi o conjunto de todas as áreas de desenvolvimento de software do IA e abrangeu as áreas chaves de acompanhamento, planejamento, requisitos, configuração e qualidade do SW-CMM nível 2 [6]. A área chave de subcontratação foi considerada não aplicável pela organização. Das noventa e nove práticas, oitenta e nove foram consideradas pontos fortes, oito pontos fracos e duas melhorias em progresso.Os resultados obtidos da mini-avaliação são apresentados no Gráfico 2.

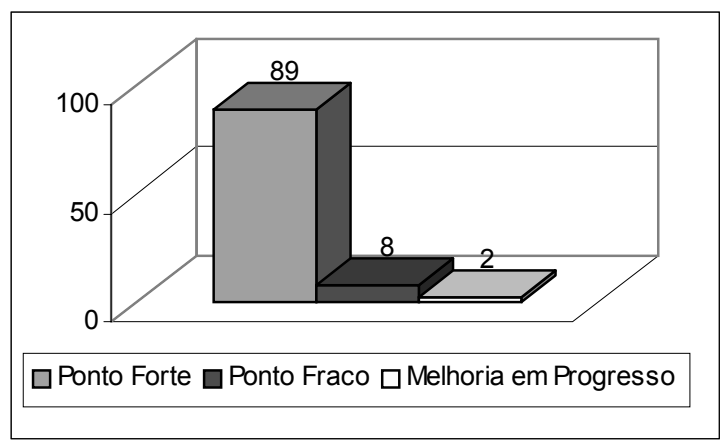

Gráfico 2: Resultado da mini-avaliação

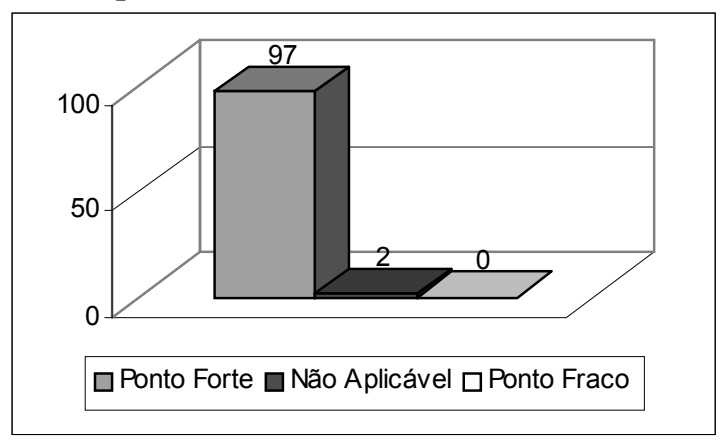

Gráfico 3: Resultado da avaliação oficial

Os problemas encontrados na mini-avaliação foram solucionados e em outubro de 2003 a avaliação oficial SW-CMM nível 2 foi realizada. Novamente, o escopo da avaliação 
foi o conjunto de todas as áreas de desenvolvimento de software do IA e abrangeu as áreas chaves de acompanhamento, planejamento, requisitos, configuração e qualidade. Das noventa e nove práticas avaliadas, noventa e sete foram consideradas pontos fortes e duas não aplicáveis para a instituição. Vale ressaltar que nenhum ponto fraco foi identificado. Os resultados obtidos na avaliação oficial estão mostrados no Gráfico 3.

\section{$4 \quad$ Resultados Obtidos}

Podemos destacar alguns dos resultados quantitativos e qualitativos obtidos com a melhoria do processo de software do IA, após a sua Certificação SW-CMM nível 2, ocorrida em outubro de 2003. O perfil dos projetos considerados para consolidar os resultados apresentados está descrito na Tabela 5.

Tabela 5: Perfil dos projetos considerados

\begin{tabular}{|l|r|l|}
\hline Duração mínima & 5 & Meses \\
\hline $\begin{array}{l}\text { Duração máxima } \\
\text { Duração média }\end{array}$ & 20 & Meses \\
\hline Esforço mínimo & 13 & Meses \\
\hline Esforço máximo & 1.500 & Homens/horas \\
\hline Esforço médio & 10.000 & Homens/horas \\
\hline Tamanho da equipe mínima & 3.200 & Homens/horas \\
\hline Tamanho da equipe máxima & 5 & pessoas \\
\hline Tamanho da equipe média & 12 & pessoas \\
\hline
\end{tabular}

Os principais resultados quantitativos estão representados nos gráficos abaixo. Os valores exibidos nesses gráficos comprovam que a qualidade nos processos do IA resultaram na qualidade dos produtos gerados. De acordo com o Gráfico 4, o número de não conformidades veio diminuindo gradativamente até chegar a um nível considerado satisfatório a partir de agosto/2003. Esse nível baixo de não conformidades permanece com a melhoria do processo de melhoria de software do IA. No Gráfico 5, a média de falhas encontradas nas releases de entrega exibem curva similar à curva de não conformidades, atingindo um número mínimo de falhas, também satisfatórios à organização.

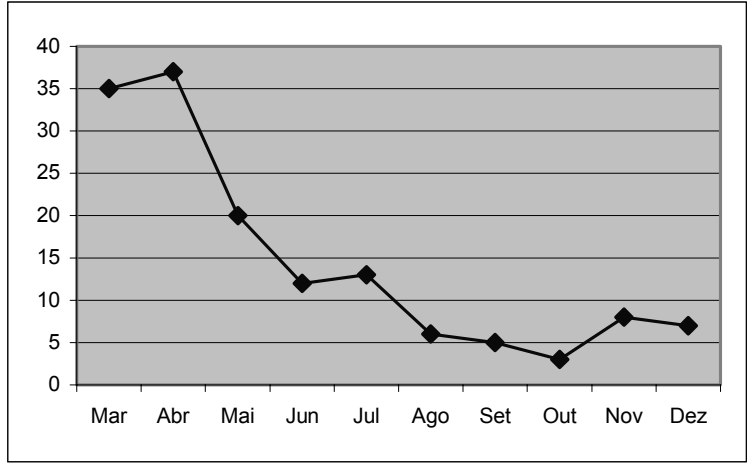

Gráfico 4: Média de não conformidades no ano de 2003

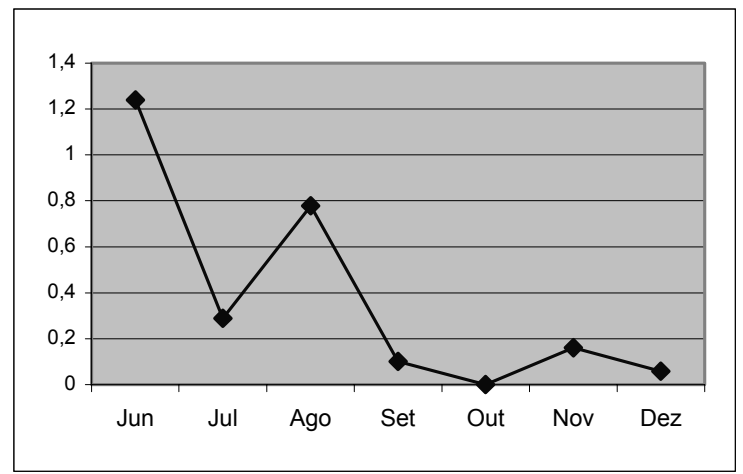

Gráfico 5: Média de falhas por casos de uso no ano de 2003

Os principais resultados qualitativos obtidos foram a definição de processos de software estruturados e maduros adequados ao IA, o estabelecimento de mecanismos para a 
melhoria desses processos, o engajamento de toda a equipe da organização, incluindo pessoas de áreas não relacionadas a software, e equipes mais comprometidas e motivadas.

\section{Conclusão}

A motivação para a realização deste trabalho foi divulgar a experiência do processo de melhoria de software do Instituto Atlântico, que culminou com sua certificação SW-CMM nível 2, mostrando os principais problemas encontrados, as soluções adotadas e os resultados obtidos.

Este artigo descreveu as principais atividades realizadas desde o planejamento e diagnóstico do processo existente até a definição e a implantação dos novos processos. Ao longo deste processo, identificamos um conjunto de lições aprendidas que devem ser destacadas:

- a participação efetiva do corpo gerencial motivou o envolvimento dos coordenadores e equipe de projeto na implantação dos processos;

- a participação de desenvolvedores no SEPG gerou uma carga indesejada para os projetos. Entretanto, os membros do SEPG atuaram como orientadores e motivadores dentro de seus projetos;

- o enfoque das avaliações de SQA teve que se adequar a fase do ciclo de vida dos projetos;

- a ênfase de atuação do grupo de SQA como orientador na condução do processo de desenvolvimento, minimiza a resistência da atuação desse grupo como avaliador, e causa empatia entre o grupo de SQA e equipe dos projetos. Além disso, em empresas de menor porte, isto pode funcionar como redutor de custos de infraestrutura de qualidade;

- a elaboração de um conjunto de procedimentos simples para os projetos, e em conformidade com as práticas-chave do SW-CMM nível 2 facilitou a institucionalização das práticas e a manutenção dos mesmos;

- a padronização dos procedimentos do SW-CMM Nível 2 para todos os projetos da organização facilitou a institucionalização e gerência dos mesmos.

Vale a pena ressaltar que as melhores práticas e métricas obtidas durante este processo pelo IA já estão compondo uma base sólida para o processo de melhoria do SW-CMM rumo ao nível 3.

\section{Referências}

[1] Almeida, M. S., Moraes, L. F. R. Qualidade de Vida no Trabalho dos Profissionais de Informática e Cultura da Qualidade nas Empresas de Informática, Workshop de Qualidade, IX SBES, Recife, 1995.

[2] Paulk, M. C. et al. Capability Maturity Model for Software, Version 1.1, Technical Report SEI-CMU-93-TR-24, Software Engineering Institute, 1993.

[3] Grady, R. D. Successful Software Process Improvement, Hewlett-Packard Book, 1997.

[4] Sommerville, Ian. Software Engineering, $6^{\text {th }}$ Edition, Addison-Wesley Publishers Ltd., 2001. ISBN 0-201-39815-X.

[5] Caputo, K. CMM Implementation Guide, Choreographing Software Process Improvement. Addison-Wesley Publishers Ltd, 1998. ISBN 0-201-37938-4

[6] Paulk, M. C. et al. Capability Maturity Model for Software, Version 1.1, Technical Report SEI-CMU-93-TR-25, Software Engineering Institute, 1993. 